\title{
Reductio ad Absurdum: Modern Physics' Incomplete Absurd Relativistic Mass Interpretation and the Simple Solution That Saves Einstein's Formula
}

\author{
Espen Gaarder Haug \\ Norwegian University of Life Sciences, Ås, Norway \\ Email: espenhaug@mac.com
}

How to cite this paper: Haug, E.G. (2018) Reductio ad Absurdum: Modern Physics' Incomplete Absurd Relativistic Mass Interpretation and the Simple Solution That Saves Einstein's Formula. Journal of Modern Physics, 9, 2623-2637.

https://doi.org/10.4236/jmp.2018.914163

Received: November 12, 2018

Accepted: December 25, 2018

Published: December 28, 2018

Copyright $\odot 2018$ by author and Scientific Research Publishing Inc. This work is licensed under the Creative Commons Attribution International License (CC BY 4.0).

http://creativecommons.org/licenses/by/4.0/

\begin{abstract}
This paper discusses an absurdity that is rooted in the modern physics' interpretation of Einstein's relativistic mass formula when $v$ is very close to $c$. Modern physics (and Einstein himself) claimed that the speed of a mass can never reach the speed of light. Yet at the same time they claim that it can approach the speed of light without any upper limit on how close it could get to that special speed. As we will see, this leads to some absurd predictions. If we assert that a material system cannot reach the speed of light, an important question is then, "How close can it get to the speed of light?" Is there a clear-cut boundary on the exact speed limit for an electron, as an example? Or must we settle for a mere approximation?
\end{abstract}

\section{Keywords}

Relativistic Mass, Maximum Velocity of Subatomic Particles, Boundary Condition, Haug Maximum Velocity

\section{Introduction}

Einstein's relativistic energy mass formula [1] [2] is given by

$$
\frac{m c^{2}}{\sqrt{1-\frac{v^{2}}{c^{2}}}}
$$

Further, Einstein commented on his own formula.

This expression approaches infinity as the velocity $v$ approaches the velocity of light $c$. The velocity must therefore always remain less than $c$, however great 
may be the energies used to produce the acceleration.

Carmichael (1913) [3] came up with a similar statement in relation to Einstein's theory:

The velocity of light is a maximum which the velocity of a material system may approach but never reach.

We certainly agree with Einstein's formula. Our question is, "How close can $v$ be to $c$ ?" Modern physics says nothing about this, except that it can approach $c$, but never reach $c$. Does this mean that one can make it as close to $c$ as one wants? This is what we will look into here, and we will show that without a more specific boundary condition on $v$ this can lead to truly absurd predictions.

Einstein's relativistic mass equation predicts that a mass will keep increasing as the velocity of the mass approaches the velocity of the speed of light. If $v=c$, then the mass would become infinite. Einstein and others have given an ad hoc solution to the problem, namely in claiming that indeed the relativistic mass never can become infinite, as this would require an infinite amount of energy for the acceleration. Still, they also seem to claim that the speed of subatomic particles can get as close to $c$ as one would want.

The discussion above is also fully relevant at today's university campus. For example, the excellent text book "University Physics" ${ }^{2}$ by Young and Freedman [4] states that

When the particle's speed $v$ is much less than $c$, this is approximately equal to the Newtonian expression...In fact as $v$ approaches $c$, the momentum approaches infinity.

Here I have marked part of the sentence in bold. Similarly, in another well-known and excellent university text book by Walker [5] we can read ${ }^{3}$.

As $v$ approaches the speed of light, the relativistic momentum becomes significantly larger than the classical momentum, eventually diverging to infinity as $v \rightarrow c$.

Similarly, in the university physics text book by Cutnell and Johnson [6] we can read"

As $v$ approaches the speed of light $c$, the $\sqrt{1-v^{2} / c^{2}}$ term in the denominator approaches zero. Hence, the kinetic energy becomes infinitely large. However, the work-energy theorem tells us that an infinite amount of work would have to be done to give the object an infinite kinetic energy. Since an infinite amount of work is not available, we are left with the conclusion that the objects with mass cannot attain the speed of light $c$.

I do not directly disagree; mathematically this is correct. My point is that modern physics does not give an exact limit on how close $v$ can get to $c$, and we will soon see how this leads to absurd relativistic masses and kinetic energies. In

${ }^{1}$ This quote is taken from page 53 in the 1931 edition of Einstein's book Relativity: The Special and General Theory. English translation version of Einstein's book by Robert W. Lawson.

${ }^{2} 14$ th edition, page 1238 .

${ }^{3}$ Fourth edition, page 1026 .

${ }^{4} \mathrm{Nin}$ th edition, page 884 . 
the otherwise excellent book on special relativity by Sartori [7] we can read ${ }^{5}$ :

According to equation (7.12), the kinetic energy of a body approaches infinity when its speed approaches $c$. This important prediction is confirmed by the experimental data.

I will claim that these statements are partly wrong, or at least they are not precise. No experiment has shown that the kinetic energy approaches infinity. What has been shown is that the kinetic energy increases rapidly as a particle is accelerated towards a velocity significantly close to the speed of light.

In 1965, Max Born [8] stated that ${ }^{6}$

A glance at Formula $(78)^{7}$ for the mass tells us that the values of the relativistic mass $m$ become greater as the velocity $v$ of the moving body approaches the speed of light. For $v=c$ the mass becomes infinitely great. From this it follows that it is impossible to make a body move with a velocity greater than that of light by applying forces: Its inertial resistance grows to an infinite extent and prevents the velocity of light from being reached.

Long ago, in 1893 Thomson [9] wrote $^{8}$

When in the limit $v=c$ the increase in mass is infinite, thus the charged sphere moving with velocity of light behaves as if its mass were infinite...

Naturally, Thomson did not know about Einstein's theory of special relativity, as it was published 12 years later. Still, his equations pointed to a similar result concerning mass when $v$ approaches $c$.

\section{The Absurdity of the Electron Following Modern Physics' Incomplete Relativistic Mass Interpretation}

An electron is a very small so-called fundamental particle with a rest-mass of approximately $m_{e} \approx 9.10938356 \times 10^{-31} \mathrm{~kg}$ (NIST CODATA 2014). Next let's look at the relativistic mass of the electron as $v$ approaches, but never reaches, the speed of light.

\section{Absurd one $\mathrm{Kg}$ mass electron}

Assume an electron is accelerated (by a giant exploding star, or by the core of a galaxy, for example) to the following velocity

$$
v=c \times 0.999999999999999999999999999999999999999
$$

That is 70 nines behind the decimal point followed by the number 586 , or we could say it is $586 \times 10^{-73}$ with nines instead of zeros after the decimal point. It gives a relativistic mass for a single electron of approximately $1 \mathrm{~kg}$.

\section{Absurd Moon mass electron}

Assume an electron is accelerated (by for example a giant exploding star, or by the core of a galaxy) to the following velocity

\footnotetext{
${ }^{5}$ Page 209.

${ }^{6}$ Page 277.

${ }^{7}$ Here Born is referring to the Einstein relativistic mass formula.

${ }^{8}$ Page 21 . Actually, Thomson used $V$ as symbol for the speed of light and $w$ for the velocity of the object. We have replaced these with $c$ and $v$ in the citation to make it easier to follow.
} 
That is 116 nines behind the decimal point followed by the number 23, or we could say it is $923 \times 10^{-118}$ with nines instead of zeros after the decimal point. It gives a relativistic mass for a single electron of approximately $7.34 \times 10^{22} \mathrm{~kg}$, that is basically equal to the rest-mass of the Moon. That is quite amazing, a tiny electron that suddenly has a relativistic mass equal to the rest-mass of the Moon! Where can we find such electrons?

\section{Absurd Earth mass electron}

Assume an electron is accelerated to the following velocity

$$
\begin{gathered}
v=c \times 0.99999999999999999999999999999999999999999999999 \\
999999999999999999999999999999999999999999999999 \\
999999999999999999999999884
\end{gathered}
$$

That is 119 nines behind the decimal point followed by the number 884 , or we could say it is $884 \times 10^{-122}$ with nines instead of zeros after the decimal point. It gives a relativistic mass for a single electron of $5.9806 \times 10^{24} \mathrm{~kg}$, that is basically equivalent to the rest-mass of the Earth.

\section{Absurd Sun mass electron \\ Assume an electron accelerated to the following velocity

$$
\begin{aligned}
& v=c \times 0.9999999999999999999999999999999999999999999999 \\
& 99999999999999999999999999999999999999999999999 \\
& 9999999999999999999999999999999999999895
\end{aligned}
$$

That is 130 nines behind the decimal point followed by the number 895 . It gives a relativistic mass for a single electron equal to the rest-mass of the Sun, that is about $1.98 \times 10^{30} \mathrm{~kg}$.

\section{Absurd Milky Way mass electron}

Assume an electron is accelerated to the following velocity

$$
\begin{aligned}
& v=c \times 0.99999999999999999999999999999999999999999999 \\
& 999999999999999999999999999999999999999999999 \\
& 999999999999999999999999999999999999999999999 \\
& 99999999999999999999895
\end{aligned}
$$

The relativistic mass of the electron at this velocity is equal to the rest-mass of the Milky Way, that is about $10^{12}$ solar masses. Still, the electron is traveling below the speed of light, so this does not go against mainstream modern physics.

\section{Insane Observable Universe electron}

Assume an electron is accelerated to the velocity of

$$
\begin{aligned}
v=c \times & 0.999999999999999999999999999999999999999999999 \\
& 9999999999999999999999999999999999999999999999 \\
& 9999999999999999999999999999999999999999999999 \\
& 99999999999999999999999999999999999996
\end{aligned}
$$

That is 174 nines behind the decimal point followed by the number 6 , or we 
could say it is $6 \times 10^{-175}$ with nines instead of zeros after the decimal point. It gives a relativistic mass for a single electron of approximately $1.018 \times 10^{52} \mathrm{~kg}$, that is basically equal to the rest-mass of what main frame physics claims is the approximate mass of the observable universe, see [10] [11] [12] [13]. That is quite amazing, a tiny electron that suddenly has a relativistic mass equal to the rest-mass of the whole observable universe.

\section{Modern physics leads to absurd kinetic energies for subatomic particles}

Table 1 lists the relativistic kinetic energy of an electron traveling at various velocities, all below the speed of light. All of these velocities are valid inside the framework of modern physics, as it stipulates no precise speed limit on the velocity of an electron as long as it falls below the speed of light.

Why don't we see a single electron (or other subatomic particle) with a relativistic mass equal to (even at the most moderate level) the rest-mass of the Moon? Such an electron would have enormous kinetic energy, causing a gigantic impact with collision with the Earth, or other planets in our solar system. We suspect that mainstream physics does not have a good answer to this question. Maybe such fast-traveling electrons exist, but they are rare and therefore have a very low probability of occurring? What if, as a counterpoint, a single electron wiped the dinosaurs out? Are we doomed? And why have we not heard physicists discussing such velocities for electrons? Perhaps they simply do not like to talk about such things, as they have no good explanations for why such very fast electrons have never been observed?

\section{A Simple Solution to the Absurdity That Saves Einstein's Relativistic Mass Formula}

We have seen how modern physics' assumption that a mass must travel more slowly than the speed of light, while at the same time asserting that it can approach the speed of light, leads to absurd predictions. An important question is, therefore, if there could be an exact speed limit below the speed of light for anything with rest-mass. Haug has recently suggested a maximum velocity for anything with rest-mass by assuming the maximum relativistic mass (energy) for

Table 1. The table shows the kinetic energy for an electron traveling at various velocities below the speed of light.

\begin{tabular}{|c|c|c|c|}
\hline $\begin{array}{l}\text { Velocity of electron } \\
\% \text { of light }(v / c) \text { : }\end{array}$ & $\begin{array}{l}\text { Relativistic electron } \\
\text { mass }=\text { rest-mass of }\end{array}$ & $\begin{array}{l}\text { Kinetic } \\
\text { energy: }\end{array}$ & $\begin{array}{l}\text { Ton TNT } \\
\text { equivalent: }\end{array}$ \\
\hline $923 \times 10^{-120} \quad$ (9'ns in front) & Moon & $6.597 \times 10^{39} \mathrm{~J}$ & $1.58 \times 10^{30}$ \\
\hline $884 \times 10^{-122} \quad$ (9'ns in front) & Earth & $5.375 \times 10^{41} \mathrm{~J}$ & $1.28 \times 10^{32}$ \\
\hline $895 \times 10^{-133} \quad$ (9'ns in front) & Sun & $1.787 \times 10^{47} \mathrm{~J}$ & $4.27 \times 10^{37}$ \\
\hline $895 \times 10^{-145} \quad$ (9'ns in front) & Milky Way & $1.787 \times 10^{59} \mathrm{~J}$ & $4.27 \times 10^{49}$ \\
\hline
\end{tabular}

${ }^{a}$ The Kinetic energy is calculated as $E_{k}=\frac{m c^{2}}{\sqrt{1-\frac{v^{2}}{c^{2}}}}-m c^{2} .{ }^{\mathrm{b}}$ One ton TNT equivalent is about 4.184 giga joules. 
an elementary particle is the Planck mass (energy), see [14]-[19]. The maximum velocity any subatomic particle can take as measured by Einstein-Poincaré synchronized clocks ${ }^{9}$ is then given by

$$
v_{\max }=c \sqrt{1-\frac{l_{p}^{2}}{\bar{\lambda}^{2}}}
$$

where $\bar{\lambda}$ is the reduced Compton wavelength of the mass in question, and $l_{p}$ is the Planck length [21] [22]. This formula can be derived by setting

$$
\begin{gathered}
m_{p} c^{2} \geq \frac{m c^{2}}{\sqrt{1-\frac{v^{2}}{c^{2}}}} \\
\sqrt{1-\frac{v^{2}}{c^{2}}} \geq \frac{m}{m_{p}} \\
1-\frac{v^{2}}{c^{2}} \geq \frac{m^{2}}{m_{p}^{2}} \\
v^{2} \leq c^{2}\left(1-\frac{m^{2}}{m_{p}^{2}}\right) \\
v \leq c \sqrt{1-\frac{m^{2}}{m_{p}^{2}}}
\end{gathered}
$$

where $m_{p}$ is the Planck mass, and $m$ is the mass of an elementary particle. And since any elementary mass can be written as

$$
m=\frac{\hbar}{\bar{\lambda}} \frac{1}{c}
$$

Which means the mass of an elementary particle can be found by measuring the Compton wavelength of the particle, as has been done experimentally with electrons, see [23]. Since the reduced Compton wavelength of the Planck mass must be the Planck length, then we must have

$$
\begin{gathered}
v \leq c \sqrt{1-\frac{\left(\frac{\hbar}{\bar{\lambda}} \frac{1}{c}\right)^{2}}{\left(\frac{\hbar}{l_{p}} \frac{1}{c}\right)^{2}}} \\
v \leq c \sqrt{1-\frac{l_{p}^{2}}{\bar{\lambda}^{2}}}
\end{gathered}
$$

The same formula can also be derived by assuming that the shortest possible length-contracted Compton wavelength is limited by the Planck length

$$
\bar{\lambda} \sqrt{1-\frac{v^{2}}{c^{2}}} \geq l_{p}
$$

${ }^{9}$ This also holds true if measured with clocks synchronized with very slow clock transportation method, see [20]. 


$$
\begin{aligned}
& 1-\frac{v^{2}}{c^{2}} \geq \frac{l_{p}^{2}}{\bar{\lambda}^{2}} \\
& v \leq c \sqrt{1-\frac{l_{p}^{2}}{\bar{\lambda}^{2}}}
\end{aligned}
$$

Further, the same maximum velocity of matter can also be found from Heisenberg's uncertainty principle when assuming the uncertainty in position cannot be smaller than the Planck length [24] [25]. So again this would mean the maximum relativistic mass of a elementary particle is the Planck mass. The Planck mass is approximately $2.17651 \times 10^{-8} \mathrm{~kg}$. It is enormous compared to the electron, but it is miniscule compared to the mass of the Moon, Earth, or the Sun. Further, the Planck mass only can last for an instant (the Planck time), as pointed out by Haug, something we soon will get back to soon.

Further, an electron can travel at a velocity very close to that of the speed of light, but its maximum velocity will still be significantly less than what has been described here previously. The maximum velocity for an electron would be approximately

$v_{\max }=c \sqrt{1-\frac{l_{p}^{2}}{\bar{\lambda}_{e}^{2}}} \approx c \times 0.999999999999999999999999999999999999999999999124$

In this calculation, we have assumed the reduced Compton wavelength of the electron given by NIST CODATA, that is $\frac{2.4263102367 \times 10^{-12}}{2 \pi} \mathrm{m}$, and a Planck length of $1.616229 \times 10^{-35} \mathrm{~m}$. Because there is some uncertainty regarding both the exact Planck length and the reduced Compton wavelength, there is some uncertainty around this velocity, but it must be very close to this number. NIST (2014) CODATA reports a standard uncertainty for the Planck length of $0.000038 \times 10^{-35} \mathrm{~m}$. Based on this theory, we can rest assured that the electron (or any other mass) can never reach a relativistic mass even close to one $\mathrm{kg}$, so there is no chance that a single electron will cause much harm (at least not compared to the data in Table 1), no matter how fast it is accelerated because there is a maximum velocity that limits both its kinetic energy and its relativistic mass.

Will modern physics accept the existence of a maximum speed limit for subatomic masses or will the field keep holding on to absurd beliefs? If we do not accept the idea that the maximum velocity for subatomic particles has an exact limit below the speed of light, then we must accept the following absurdities:

- That there is a wavelength shorter than the Planck length.

- That there is a time interval shorter than the Planck time.

- That there is a maximum frequency higher than the Planck frequency.

- That an electron can take a relativistic mass similar to that of the Moon, the Earth, the Sun, and even the Milky Way, or even larger masses. This is, at best, truly absurd! Our theory predicts that no subatomic particle can take a relativistic mass higher than the Planck mass. 
- That there is no limit on the relativistic Doppler shift. This is also highly unlikely. Haug [15] has suggested that the limit here is the Planck frequency Doppler shift.

- For a subatomic particle, there is a momentum close to infinity. This is absurd. The maximum momentum of a subatomic particle based on our maximum velocity formula for matter is likely just below the Planck momentum.

- For a subatomic particle, there is a kinetic energy close to infinity. This is, again, absurd.

The newly introduced maximum velocity puts a series of limits on subatomic "fundamental particles":

- The maximum frequency is the Planck frequency: $f_{\max }=\frac{c}{l_{p}}$.

- The maximum relativistic mass a subatomic particle can take is the Planck mass.

- The maximum relativistic momentum a subatomic particle can take is just below the Planck momentum.

- The maximum kinetic energy a subatomic particle can take is close to $\frac{\hbar}{l_{p}} c$, or more precisely $E_{k, \max }=\hbar c\left(\frac{1}{l_{p}}-\frac{1}{\bar{\lambda}}\right)$.

- The maximum relativistic length contraction of the reduced Compton wavelength a subatomic particle is $l_{p}$, which is the reduced Compton length of the Planck mass particle. This again means the maximum relativistic mass of a elementary particle is the Planck mass.

Also, it is worth mentioning here that the Planck length can be found totally independent of any knowledge of Newton's gravitational constant, see [18] and even independent of any knowledge of the Planck constant, see [26].

\section{Ways to Write the Maximum Velocity Formula}

There are several ways to write the maximum velocity for subatomic particles that will all give the same answer; here we present some of them.

In terms of reduced Compton wavelength

$$
v_{\max }=c \sqrt{1-\frac{l_{p}^{2}}{\bar{\lambda}^{2}}}
$$

In terms of particle mass

$$
v_{\max }=c \sqrt{1-\frac{m^{2}}{m_{p}^{2}}}
$$

where $m$ is the rest-mass of the particle and $m_{p}$ is the Planck mass.

As a function of Newton's gravitational constant

$$
v_{\max }=c \sqrt{1-\frac{G m^{2}}{\hbar c}}
$$


All of these formulas are basically the same, but each one requires somewhat different input:

$$
v_{\max }=c \sqrt{1-\frac{l_{p}^{2}}{\bar{\lambda}^{2}}}=c \sqrt{1-\frac{m^{2}}{m_{p}^{2}}}=c \sqrt{1-\frac{G m^{2}}{\hbar c}}
$$

\section{The maximum velocity for an electron}

For an electron, the maximum velocity can be written as function of the dimensionless gravitational coupling constant.

$$
v_{\max }=c \sqrt{1-\alpha_{G}}
$$

This is no surprise, since the dimensionless gravitational coupling constant is given by $\alpha_{G}=\frac{m_{e}^{2}}{m_{p}^{2}}=\frac{l_{p}^{2}}{\bar{\lambda}_{e}^{2}}$. For information about the dimensionless gravitational coupling constant see [27] [28] [29] [30].

\section{The Speed of the Planck Mass Particle}

In the maximum velocity formula given by Haug, the maximum speed of any observed particle is very close to light, but still faster than what we can achieve at our strongest particle accelerators such as the Large Hadron Collider. However, in the special case where we deal with a Planck mass particle then the reduced Compton wavelength is equal to the Planck length, $\bar{\lambda}=l_{p}$ and we must have

$$
v_{\max }=c \sqrt{1-\frac{l_{p}^{2}}{l_{p}^{2}}}=0
$$

That the maximum velocity of the Planck mass particle is zero sounds bizarre at first. However, as suggested by [17] the Planck mass particle is the collision point between two photons. Light always travel at the speed of light, but what is the speed of a photon just at the moment it collides with another photon? We will claim a photon stands still just at the instant (Planck time) it collides with another photon. Recent research has been quite clear on the concept that in a photon-photon collision we likely can create matter, see [31]. We will claim the collision point between two light particles is the missing Planck mass particle. The Planck mass energy $E=p_{p} c=m_{p} c c$ seems far too great for any observed photons. However, in our analysis, we claim that the Planck mass particle only can last for one Planck second: $t_{p}=\frac{l_{p}}{c}$, before it dissolves into energy and where the light particles once again travel at the speed of light. This means the minimum momentum of a photon is

$$
m_{p} c \frac{l_{p}}{c}=\frac{\hbar}{c}
$$

and multiplied by $c$ we get the energy of the light particle, that is $\hbar$, and dividing this by $c^{2}$ we get 


$$
\frac{\hbar}{c^{2}}=m_{p} t_{p}
$$

Haug has recently suggested that all masses ultimately consist of Planck mass particles, but again these Planck mass particle events only last for one Planck second. Schrödinger in 1930 indicated that the electron was in a sort of Zitterbewegung ("trembling motion" in English) $\frac{2 m c^{2}}{\hbar} \approx 1.55269 \times 10^{21}$ per second. We will suggest that the electron is in a Planck mass state $\frac{c}{\bar{\lambda}_{e}} \approx 7.76344 \times 10^{20}$ per second (about half of that of Schrödinger's "Zitterbewegung" frequency). However, each Planck mass state only lasts for one Planck second and therefore we get the normal electron mass from

$$
\frac{c}{\bar{\lambda}_{e}} m_{p} \frac{l_{p}}{c}=\frac{\hbar}{\bar{\lambda}_{e}} \frac{1}{c} \approx 9.10938 \times 10^{-31} \mathrm{~kg}
$$

That is to say, every elementary particle is also a clock ticking at the reduced Compton periodicity. The idea of an internal clock with a clock frequency close to the "Zitterbewegung" frequency in the case of the electron is not new, see [32] [33], for example. The link between mass and Compton time frequency has recently been supported by recent experimental research. Dolce and Perali [34] conclude that "the rest-mass of a particle is associated to a rest periodicity known as Compton periodicity". Again, in our model the reduced Compton periodicity is directly linked to a Planck mass event. Between each Planck mass event there is a reduced Compton time interval: $\frac{\bar{\lambda}}{c}$. The Planck mass event itself we suggest only lasts for one Planck second and that even an elementary particle is in an internal energy state most of the time; only in the time fraction $\frac{l_{p}}{\bar{\lambda}}$ is the particle in a Planck mass state. The rest of the time it is in an internal energy state. An electron switches between energy and mass state approximately $7.76 \times 10^{20}$ times per second.

This would mean that the mass of an elementary particle such as an electron is time dependent. However, evaluating the time dependency of elementary particles can likely only be done directly when one measures the mass of an elementary particle at time windows close to the reduced Compton time of the particle, where the reduced Compton time simply is defined as the reduced Compton wavelength of the particle divided by the speed of light. This time interval is so short that we are not really able to complete such a study yet, even for an electron.

In 1899, Max Planck was the first to suggest that there was an important elementary mass given by $m_{p}=\sqrt{\frac{\hbar c}{G}}$. He derived the Planck mass using dimensional analysis, assuming that the Newton gravitational constant, the Planck constant, and the speed of light were the most important universal con- 
stants. It was Lloyd Motz, while working at the Rutherford Laboratory in 1962, [35] [36] [37] who first suggested that there likely existed a very fundamental particle with a mass equal to the Planck mass that he called the "Uniton." Motz acknowledged that his Unitons (Planck mass particles) had far too much mass compared to known subatomic masses. The Planck mass one gets from the Max Planck formula is approximately $2.176 \times 10^{-8} \mathrm{~kg}$. Motz tried to explain this by claiming that the Unitons had radiated most of their energy away:

According to this point of view, electrons and nucleons are the lowest bound states of two or more Unitons that have collapsed down to the appropriate dimensions gravitationally and radiated away most of their energy in the process. -Lloyd Motz

Others have suggested that there were plenty of Planck mass particles around just after the Big Bang, see [38], but that most of the mass of these super-heavy particles has radiated away. Several physicists, including Motz and Hawking, have suggested that such particles could be micro black holes [39] [40] [41]. Planck mass particles have even been proposed as candidates for cosmological dark matter [42] [43].

Still, no sign of the enormous Planck mass particle has been found, and there have been no observations of micro black holes yet either. The Compton clock model of matter seems to give a possible simple solution to this puzzle. In our model of matter, one needs to consider the observational time window, in particular when it comes to the Planck mass particle. The Planck mass particle likely only lasts for one Planck second. So, if observed inside one Planck second its mass is indeed its well-known Planck mass, and it is then very large compared to any observed elementary particles so far. However, we are not even close to observing particles at the Planck time scale. In a longer time window, one second for example, the Planck mass particle is only approximately $1.173 \times 10^{-51} \mathrm{~kg}$. That is, we have likely looked for a much too large mass in our search for the Planck mass particle and micro black holes. With that in mind, we should perhaps change the perspective on what a micro black hole is. In our view, it is nothing more than the very collision point between two photons: they collide and the collision lasts for one Planck second. At collision the photons stand absolutely still, and then dissolve into energy again moving at the speed of light. The micro black hole is, in this way, almost a misnomer. Further theoretical and practical research is needed to decide if this is the case or not. In particular, research looking for possible breaks in Lorentz symmetry could be important here and is what we come to in the next section.

\section{Breakdown of Lorentz Invariance at the Planck Scale?}

The maximum velocity formula for anything with rest-mass would mean Lorentz invariance breaks down at the Planck scale. Based on this view, the Planck particle, the Planck length, and the Planck time, unlike any other particle, length, or time, seem to be the same no matter what frame they are observed from. The 
view that Lorentz invariance could be broken at the Planck scale appears to be consistent with what is predicted by several quantum gravity theories, see for example [44]. Lorentz symmetry is supported by a long series of tests, but it has never been tested at anything even close to the Planck scale (at distances close to the Planck length, or Planck energies), so one should be careful to use experimental evidence as an argument against this idea.

One could ask to what degree new physics at the Planck scale could be weakly detected at lower energies; this is discussed by [45] [46], for example. A recent review article [47] on the possibility for Lorentz symmetry breaking in relation to quantum gravity predictions and experiments noted:

In conclusion, though no violation of Lorentz symmetry has been observed so far, an incredible number of opportunities still exist for additional investigations.

So, we think testable predictions related to the Planck scale should be investigated further, also in relation to our maximum velocity formula. Recently, Haug [48] has indicated that this new maximum velocity of matter likely predicts zero velocity time-dilation in quasars, which is consistent with what has been observed [49] [50]. The Schwarzschild radius is linked to the Planck length, $R_{s}=2 \frac{G m}{c^{2}}=2 N \frac{l_{p}^{2}}{\bar{\lambda}}$, where $N$ is the number of Planck masses in the mass in question. This, combined with the fact that quasars are often considered black holes (at least at their core), means quasars likely are linked to the Planck scale. It could be that we already have observations from the Planck scale from quasars, and that such anomalies as lack of time dilation in quasars can distinguish various theories in relation to their prediction of how Lorentz symmetry is broken down or not broken down. At least it seems that our theory in relation to this maximum velocity likely is consistent with everything that has been observed so far.

\section{Conclusion}

We conclude that in stating that a mass must travel more slowly than the speed of light, while at the same time asserting that it can approach the speed of light, we get absurd predictions. Examples include the idea that an electron could attain a relativistic mass equal to the rest-mass of the Moon, the Earth, the Sun, the Milky Way, or even entire galaxy clusters. Haug has recently addressed this absurdity by showing that there must be a precise maximum velocity for anything with mass likely given by $v_{\max }=c \sqrt{1-\frac{l_{p}^{2}}{\bar{\lambda}^{2}}}$.

\section{Acknowledgements}

Thanks to Victoria Terces for helping me edit this manuscript. Also thanks to Alan Lewis, Daniel Duffy, ppauper, and AvT for useful tips on how to do high precision calculations. Also thanks to an anonymous referee for very useful comments. 


\section{Conflicts of Interest}

The author declares no conflicts of interest regarding the publication of this paper.

\section{References}

[1] Einstein, A. (1905) Annalen der Physik, 323, 639-641. https://doi.org/10.1002/andp.19053231314

[2] Einstein, A. (1916) Relativity: The Special and the General Theory. Translation by Robert Lawson (1931), Crown Publishers, New York.

[3] Carmichael, R.D. (1913) The Theory of Relativity. John Wiley \& Sons, Hoboken.

[4] Freedman, R.A. and Young, H.D. (2016) University Physics with Modern Physics, 14th Edition, Pearson, Boston.

[5] Walker, J.S. (2010) Physics. 4th Edition, Addison-Wesley, Boston.

[6] Cutnell, J.D. and Johnson, K.W. (2012) Physics. 9th Edition, John Wiley \& Sons, Inc., Hoboken.

[7] Sartori, L. (1996) Understanding Relativity. University of California Press, Oakland.

[8] Born, M. (1965) Einstein's Theory of Relativity. Dover, Mineola, New York.

[9] Thomson, J.J. (1893) Recent Researches in Electricity and Magnetism. Oxford University Press, Oxford.

[10] Whitrow, G.J. (1946) Nature, 185, 165-166. https://doi.org/10.1038/158165b0

[11] Dirac, P. (1947) The Principles of Quantum Mechanics. Oxford University Press, Oxford.

[12] Carvalho, J.C. (1995) International Journal of Theoretical Physics, 34, 2507-2509. https://doi.org/10.1007/BF00670782

[13] Persinger, M.A. (2009) Journal of Physics, Astrophysics and Physical Cosmology, 3, $1-3$.

[14] Haug, E.G. (2016) The Planck Mass Particle Finally Discovered! Good Bye to the Point Particle Hypothesis! http://vixra.org/pdf/1607.0496v7.pdf

[15] Haug, E.G. (2016) A New Solution to Einstein's Relativistic Mass Challenge Based on Maximum Frequency. http://vixra.org/pdf/1609.0083v2.pdf

[16] Haug, E.G. (2016) Physics Essays, 29, 558-561.

[17] Haug, E.G. (2017) Acta Astronautica, 136, 144-147. https://doi.org/10.1016/j.actaastro.2017.03.011

[18] Haug, E.G. (2017) Applied Physics Research, 9, 58.

[19] Haug, E.G. (2018) International Journal of Astronomy and Astrophysics, 8, 6-23. https://doi.org/10.4236/ijaa.2018.81002

[20] Haug, E.G. (2014) Unified Revolution: New Fundamental Physics. E.G.H. Publishing, Oslo.

[21] Planck, M. (1899) Naturlische Masseinheiten. Der Königlich Preussischen Akademie Der Wissenschaften, Berlin, 479.

[22] Planck, M. (1906) Vorlesungen über die Theorie der Wärmestrahlung. J.A. Barth, Leipzig, 163.

[23] Prasannakumar, S., Krishnaveni, S. and Umesh, T.K. (2012) European Journal of Physics, 33, 65-72. https://doi.org/10.1088/0143-0807/33/1/005 
[24] Haug, E.G. (2018) Does Heisenberg's Uncertainty Principle Predict a Maximum Velocity for Anything with Rest-Mass below the Speed of Light? http://vixra.org/pdf/1801.0218v4.pdf

[25] Haug, E.G. (2018) Revisiting the Derivation of Heisenberg's Uncertainty Principle: The Collapse of Uncertainty at the Planck Scale. https://doi.org/10.20944/preprints201805.0258.v2

[26] Haug, E.G. (2018) Finding the Planck Length Independent of Newton's Gravitational Constant and the Planck Constant: The Compton Clock Model of Matter. https://www.preprints.org/manuscript/201809.0396/v1

[27] Silk, J. (1977) Nature, 265, 710-711. https://doi.org/10.1038/265710a0

[28] Rozental, I.L. (1980) Soviet Journal of Experimental and Theoretical Physics Letters, 31, 19-27.

[29] Neto, M.D.O. (2005) Chaos, Solitons and Fractals, 24, 19-27. https://doi.org/10.1016/j.chaos.2004.07.032

[30] Burrows, A.S. and Ostriker, J.P. (2013) Astronomical Reach of Fundamental Physics. Proceedings of the National Academy of Sciences of the United States of America, 111, 31-36.

[31] Pike, O.J., Mackenroth, F., Hill, E.G. and Rose, S.J. (2014) Nature Photonics, 8, 434-436. https://doi.org/10.1038/nphoton.2014.95

[32] Rivas, M. (2008) Measuring the Internal Clock of the Electron.

[33] Bauer, M. (2014) Electron Channeling, de Broglie's Clock and the Relativistic Time Operator.

[34] Dolce, D. and Perali, A. (2015) The European Physical Journal Plus, 130, 41. https://doi.org/10.1140/epjp/i2015-15041-5

[35] Motz, L. (1962) Il Nuovo Cimento, 26, 672-697. https://doi.org/10.1007/BF02781795

[36] Motz, L. (1966) A Gravitational Theory of the mu meson and Leptons in General. Rutherford Observatory, Columbia University, New York.

[37] Motz, L. (1971) The Quantization of Mass. Rutherford Observatory, Columbia University, New York.

[38] De, S.S. (2001) International Journal of Theoretical Physics, 40, 2067-2084. https://doi.org/10.1023/A:1011953623319

[39] Hawking, S. (1971) Monthly Notices of the Royal Astronomical Society, 152, 75-78. https://doi.org/10.1093/mnras/152.1.75

[40] Motz, L. and Epstein, J. (1979) Il Nuovo Cimento, 51, 88-113. https://doi.org/10.1007/BF02822327

[41] Obermair, G.M. (2013) Journal of Physics, Conference Series, 442, Article ID: 012066. https://doi.org/10.1088/1742-6596/442/1/012066

[42] Macgibbon, J.H. (1987) Nature, 329, 308-309. https://doi.org/10.1038/329308a0

[43] Dokuchaev, V.I. and Eroshenko, Y.N. (2014) Black Hole Atom as a Dark Matter Particle Candidate. https://arxiv.org/abs/1403.1375 https://doi.org/10.1155/2014/434539

[44] Kislat, F. and Krawczynski, H. (2017) Physical Review D, 95, Article ID: 083013. https://doi.org/10.1103/PhysRevD.95.083013

[45] Amelino-Camelia, G., Ellis, B.J., Mavromatos, N.E., Nanopoulos, D.V. and Sarkar, S. (2998) Nature, 393, 763-765. https://doi.org/10.1038/31647 
[46] Reyes, C.M., Ossandon, S. and Reyes, C. (2005) Physics Letters B, 746, 190-193. https://doi.org/10.1016/j.physletb.2015.05.006

[47] Hees, A., et al. (2017) Universe, 2, 30. https://doi.org/10.3390/universe2040030

[48] Haug, E.G. (2018) Maximum Velocity for Matter in Relation to the Schwarzschild Radius Predicts Zero-Time Dilation for Quasars.

[49] Hawkings, M.R.S. (2000) Monthly Notices of the Royal Astronomical Society, 406, 1047-1054.

[50] Hawkings, M.R.S. (2001) The Astrophysical Journal, 553, 97-100. https://doi.org/10.1086/320683 